\title{
Mechanism-based management for mucositis: option for treating side effects without compromising the efficacy of cancer therapy
}

This article was published in the following Dove Press journal:

OncoTargets and Therapy

5 April 2016

Number of times this article has been viewed

\section{Youngjoo Kwon}

Department of Food Science and Engineering, Ewha Womans University, Seoul, South Korea
Correspondence: Youngjoo Kwon Department of Food Science and Engineering, Ewha Womans University, 52 Ewhayeodae-gil, Seodaemun-gu, Seoul 03760, South Korea

Email youngjoo.kwon@ewha.ac.kr

\begin{abstract}
Mucositis is a major side effect induced by chemotherapy and radiotherapy. Although mucositis is a leading cause of morbidity and mortality in cancer patients, management is largely limited to controlling symptoms, and few therapeutic agents are available for treatment. Since mucositis could be inhibited by the modulation of radiotherapy- or chemotherapy-induced pathways independently of cancer treatment, there is an opportunity for the development of more targeted therapies and interventions. This article examined potential therapeutic agents that have been investigated for the prevention and/or inhibition of mucositis induced by conventional chemotherapy and radiotherapy. They can be classified according to their mechanisms of action: scavenging reactive oxygen species, inhibition of specific cytokine production or inflammation, and inhibition of apoptosis. These early events may be good target pathways for preventing the pathogenesis of mucositis. Considering that both cancer therapy and therapeutic agents for mucositis act on both normal and cancer cells, agents that inhibit mucositis should act through mechanisms that selectively protect normal cells without compromising cancer treatment. Therefore, mechanism-based guidance for the treatment of mucositis is critical to prevent risky treatments for cancer patients and to relieve detrimental side effects effectively from cancer therapy.
\end{abstract}

Keywords: apoptosis, chemotherapy, cytokine, mucositis, radiotherapy, reactive oxygen species

\section{Introduction}

Chemotherapy and radiotherapy are mainstay regimens for cancer treatments. However, both types of cancer treatment also affect normal cells, and their side effects on highly proliferative tissues have significant problems. One of these adverse effects is mucositis, a painful inflammation and ulceration of the mucous membrane lining the gastrointestinal tract (GIT). ${ }^{1}$ Mucositis can affect the entire mucosal lining of the GIT, but the oral and oropharyngeal mucosa are common sites. ${ }^{2,3}$

The prevalence and severity of mucositis vary according to the presence of risk factors (eg, age, sex, and certain gene types) derived from patients. ${ }^{4,5}$ The type of treatment administered also affects the incidence of mucositis. Mucositis predominantly $(60 \%-100 \%)$ occurs in patients undergoing radiotherapy, high-dose chemotherapy, and bone marrow transplantation. ${ }^{6-8}$ An estimated $40 \%$ of patients that receive standarddose chemotherapy develop mucositis. ${ }^{9}$ Conventional chemotherapeutic drugs most frequently associated with mucositis include antimetabolites, such as 5-fluorouracil (5-FU), methotrexate, and purine antagonists. ${ }^{10}$ Anthracycline antitumor antibiotics (eg, doxorubicin) and taxanes (eg, paclitaxel and docetaxel) are other chemotherapeutic drugs that commonly cause mucositis. ${ }^{10,11}$ Other treatment-related risk factors 
include dose, chemotherapy schedule, route of administration, and concomitant use of chemotherapy and radiation. ${ }^{12}$

Mucositis induced by targeted cancer therapies has not been well documented. ${ }^{13,14}$ This is probably because the side effects to normal cells receive less consideration in targeted therapies than conventional chemotherapy. ${ }^{13}$ In addition, targeted agents are often administered in conjunction with or after conventional chemotherapy treatment, making it difficult to identify toxicity exclusively derived from the targeted therapy. ${ }^{12}$ Among these, mTOR inhibitors (eg, rapamycin, everolimus, and temsirolimus) have been often associated with mucositis. ${ }^{15-17}$ Significant incidence of mucositis has been reported with some EGFR inhibitors (eg, bevacizumab and erlotinib) and tyrosine-kinase inhibitors (eg, sorafenib and sunitinib). ${ }^{15,18}$ However, the clinical presentation of targeted therapy-induced mucositis is quite different from radiation or conventional chemotherapy-induced mucositis. ${ }^{19}$ Mucositis derived from targeted chemotherapy is also less severe than that caused by conventional chemotherapy. ${ }^{19}$ It is likely that targeted therapeutics induce mucositis through different mechanisms than the pathways described for conventional chemotherapy agents, although the mechanism through which targeted agents induce mucositis is not well understood. ${ }^{12,19}$

Mucositis is a leading cause of dosage reduction and premature cessation of treatment for both chemotherapy and radiotherapy, and thus greatly impacts the survival of patients from cancer. ${ }^{11}$ Patients with mucositis exhibit severe clinical symptoms, including pain derived from ulceration, nausea, vomiting, heartburn, diarrhea, constipation, subsequent malnutrition, and weight loss. ${ }^{1,20}$ Ulceration is commonly associated with a high risk of systemic infection. ${ }^{1}$ Therefore, mucositis is a major clinical and economic burden that severely affects patient outcomes and quality of life, in addition to increasing the risk of morbidity and mortality. ${ }^{1}$ However, currently the management of mucositis is largely limited to the control of pain, oral hygiene, infection, bleeding, and malnutrition. ${ }^{8,13}$

Mucositis was considered to be merely the consequence of direct toxicity of chemotherapy and radiotherapy on rapidly dividing epithelial cells. ${ }^{1}$ However, it has been recognized that mucositis is the result of complex and multifaceted biological events involving multiple signaling pathways and interactions between the epithelium and the underlying submucosa. ${ }^{1,13}$ The idea that mucositis could be inhibited by indirect modulation of radiotherapy- or chemotherapy-initiated pathways provides an opportunity for the development of more targeted therapies and interventions. ${ }^{13,21}$
This article examines potential therapeutic agents that have been studied for the prevention and/or inhibition of mucositis induced by conventional chemotherapy and radiotherapy according to their mechanisms of action. This review suggests molecular pathways that can be targeted to inhibit the pathogenesis of mucositis, and discusses the possibility of mechanism-based management options for mucositis, as well as factors that should be considered for mucositis treatment.

\section{Pathobiology of mucositis}

Although the development of mucositis involves a complex and dynamic array of biological events, the progression of mucositis is often described in five stages: initiation, primary damage response, signal amplification, ulceration, and healing. ${ }^{3}$ It has been discovered that mucositis involves not only epithelial cells but also submucosa, supporting connective tissues that consist of fibroblasts, immune cells, blood cells, and extracellular matrices. ${ }^{3}$ This is reflected in the five-stage model revised from the previously proposed four-stage model. ${ }^{22}$

The initiation stage occurs immediately after the administration of radiation or chemotherapy. In this stage, DNA damage and mitochondria-dependent generation of reactive oxygen species (ROS) are induced by chemotherapy or radiotherapy. ${ }^{23-26}$ Cancer therapy directly damages DNA and causes strand breaks that result in the death of a small fraction of basal and suprabasal epithelial cells. ${ }^{1}$ A more pronounced effect is believed to be derived from the generated ROS, since they are also important mediators of downstream events that drive tissue damage. ${ }^{23,24}$

DNA damage and ROS generation lead to the second stage, the primary damage response. DNA-strand breaks and ROS trigger a series of interacting biological events through the activation of various transcription factors. ${ }^{1}$ $\mathrm{NF}-\kappa \mathrm{B}$ is among the most studied in relation to mucositis, and its activation increases the transcription of genes known to be associated with the progression of mucositis. ${ }^{20}$ These genes include proinflammatory cytokines (eg, IL-1 $\beta$, IL-6, TNF $\alpha$ ) and antioxidant enzymes (eg, mitochondria superoxide dismutase [SOD]). ${ }^{20,27}$ The effect of chemotherapy and radiation insult on submucosa is also suggested to occur during this stage. Although much is unknown about the effect of chemotherapy or radiation on submucosa, cytokines induced by cancer treatment may promote the secretion of destructive metalloproteases and inflammatory cytokines by submucosal fibroblasts. ${ }^{28}$ In addition, the exposure of fibroblasts to chemotherapy and radiation induces cell 
senescence, ${ }^{29}$ leading to senescence-associated secretory phenotypes characterized by the secretion of interleukins, chemokines, growth factors, proteases, and shedding of membrane-associated proteins. ${ }^{30}$

During the signal-amplification stage, the initial activation of transcription factors results in the upregulation of a broad range of effectors, including cytokines, which leads to the activation of parallel signaling pathways, amplifying the initial signals toward tissue destruction. ${ }^{1}$ In the ulceration stage, clinical manifestations of mucositis become apparent as mucosal integrity is lost and painful lesions are formed. ${ }^{1}$ The breach in the submucosa allows for the entry of resident microorganisms and bacterial colonization. ${ }^{1}$ Proinflammatory cytokine production is further induced due to this secondary infection. ${ }^{3,13}$ The patient experiences significant pain, and the risk of systemic infection increases at this stage. ${ }^{7}$ The final healing phase occurs after the cessation of cancer therapy. During this phase, signals from the submucosal extracellular matrices and mesenchyme induce reepithelialization. ${ }^{3,13}$ This phase results in the restoration of normal mucosal appearance at the clinical level. ${ }^{7}$ Figure 1 describes the pathways that have been shown to be effective for treating mucositis using potential therapeutic agents for mucositis management. These pathways exist in early events during the pathogenesis of mucositis, and thus provide good opportunities to prevent and inhibit the development of mucositis.

\section{Scavenging of ROS}

ROS act as secondary messengers in cell signaling, and are required for various biological processes in normal cells. ${ }^{31,32}$ However, excessive amounts of ROS can induce oxidative

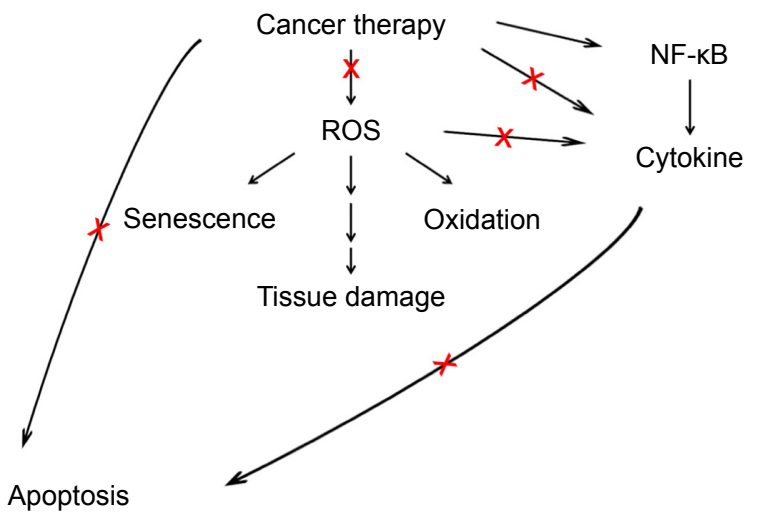

Figure I Potential pathways that can be effectively targeted for mucositis management.

Notes: Pathways are shown to be effective for treating mucositis using potential therapeutic agents for mucositis management. These pathways exist in early events during the pathogenesis of mucositis, and thus provide good opportunities to prevent and inhibit the development of mucositis.

Abbreviation: ROS, reactive oxygen species. damage to cellular molecules, including lipids, proteins, and DNA, contributing to cell death. ${ }^{33}$ Many chemotherapy and radiation treatments induce the generation of ROS that are directly toxic to mucosal cells. In addition, ROS also initiate a cascade of events that lead to tissue damage. ${ }^{23,24}$ Therefore, scavenging of the ROS induced by chemotherapy or radiotherapy could effectively prevent the initial step of the tissue-damage event.

The cellular ROS level is controlled by a balance between ROS generation and their elimination by antioxidant systems consisting of antioxidant enzymes (eg, glutathione peroxidase, glutathione reductase, SOD, and catalase) and endogenous antioxidants (eg, glutathione). Many studies demonstrated that the enhancement of antioxidative activity protects against radiation-induced mucositis. ${ }^{34-36}$ Mice administered with human Mn-SOD (SOD2) before irradiation exhibited a decrease in mucositis compared to mice with a control gene. ${ }^{34}$ Rapamycin, an mTOR inhibitor, unexpectedly induces Mn-SOD expression, leading to a decrease in $\gamma$-irradiation-induced premature senescence in normal oral keratinocytes in vitro, as well as the protection of normal cells from radiation-induced depletion of tissue-repopulating stem cells, with a reduction in ulcers and mucositis in vivo. ${ }^{35}$ Furthermore, rapamycin treatment inhibits the release of multiple senescence-associated cytokines derived from irradiated cells by preventing senescence, ${ }^{35}$ thereby further inhibiting the progression of mucositis. However, this study did not address rapamycin-induced mucositis, which was previously reported to cause mucositis. ${ }^{15-17}$ Administration of the SOD mimetic M40403 also reduced the severity and duration of mucositis in a hamster model where oral mucositis was induced by irradiation of the cheek pouch. ${ }^{36}$ In the same study, the dosage schedule was critical for the efficacy of the drug: dosing of M40403 on the day of irradiation was more effective than an extended dosage regimen. ${ }^{36}$ This result may suggest that the action of SOD is important at the time of irradiation to prevent oral mucositis, as ROS are damaging agents or initiators of signaling cascades that lead to tissue damage.

The use of antioxidants also effectively reduces mucositis through a direct antioxidative effect or by enhancing endogenous antioxidative enzymes. In the human oral epithelial cell line, RT7, $\gamma$-tocotrienol suppresses the 5-FU-induced generation of ROS. ${ }^{37}$ This is achieved by the stabilization of 5-FU-induced activation of Nrf2, a transcription factor that upregulates antioxidant enzymes (eg, heme oxygenase I and NADH:quinone oxidoreductase 1). ${ }^{37}$ Vitamin E delivered intramuscularly also delays the onset and severity of 
radiation-induced oral mucositis in rats. ${ }^{38}$ Administration of vitamin $\mathrm{E}$ restores the activity of plasma SOD and catalase that is suppressed by radiation. ${ }^{38}$ Studies also suggested that vitamin $\mathrm{E}$ is effective in treating mucositis in humans. Topical treatment with vitamin E effectively reduces oral mucositis in patients receiving chemotherapy. ${ }^{39,40}$ However, oral administration of vitamin $\mathrm{E}$ did not noticeably improve chemotherapyinduced mucositis. ${ }^{39,40}$ Supplementation with oral vitamin $E$ also had no effect on mucositis (incidence or severity of mucositis) in patients with leukemia who were undergoing bone marrow transplantation in a randomized double-blind placebo-controlled clinical trial. ${ }^{41}$ Therefore, systemic absorption of vitamin E might be poor and the route of administration may be critical for the effectiveness of vitamin $\mathrm{E}$ in treating mucositis. The glutathione precursor $N$-acetyl-L-cysteine (NAC) reduced the incidence of severe (grade 3/4) mucositis in a double-blind, randomized placebo-controlled study where leukemia patients were treated with NAC from the day of starting high-dose chemotherapy until 15 days after stem cell transplantation. ${ }^{42}$ Therefore, studies using antioxidant enzymes or small-molecule ROS scavengers have reinforced the idea that ROS are an important early trigger leading to mucositis.

ROS also play a role in inflammation. ${ }^{43}$ ROS are suggested to mediate radiotherapy- or chemotherapy-induced inflammation. ${ }^{3}$ SOD derived from bovine, known as orgotein, is also an anti-inflammatory agent and has been used for the amelioration of radiation-induced side effects. ${ }^{44}$ ROS may also promote inflammation by activating multiprotein cytoplasmic complexes called inflammasomes. ${ }^{45,46}$ For example, mucositis induced by irinotecan, a topoisomerase I inhibitor, is mediated by inflammasome activation as a result of NOX2-derived ROS generation and inflammasome-dependent production of IL-1 $\beta$ and IL-18. ${ }^{46}$ This was verified by NOX inhibition using mice deficient of gp91phox and a NOX inhibitor - apocynin. ${ }^{46}$ Therefore, the reduction of cellular ROS levels may provide an additional beneficial effect of suppressing inflammation that can accelerate the pathogenesis of mucositis. Nevertheless, no clinical practice guideline for mucositis by the Multinational Association of Supportive Care in Cancer and International Society of Oral Oncology (MASCC/ISOO) is available for many antioxidants, including orgotein and vitamin $\mathrm{E}$, due to inadequate and/or conflicting evidence. ${ }^{47}$ A suggestion is made in favor of an intervention of oral zinc supplementation to prevent oral mucositis. ${ }^{47-49}$ Zinc has antioxidant properties. ${ }^{50}$ However, it is unknown how zinc plays an inhibitory role in mucositis.

There is however considerable concern that antioxidants may adversely affect the efficacy of cancer treatment. ${ }^{33,51,52}$
Concurrent administration of two different free radical scavengers, vitamin $\mathrm{E}$ and $\beta$-carotene, with radiotherapy for head and neck cancer modestly decreases acute toxicity. ${ }^{53}$ However, this regimen increases tumor recurrence and second primary tumors in head and neck cancer patients. ${ }^{53,54}$ Dietary NAC and vitamin E markedly increased tumor progression and reduced survival in mouse models of B-Raf- and K-Rasinduced lung cancer. ${ }^{55}$ Lanperisone induces ROS in cells that harbor the KRAS mutation, a frequent oncogenic mutation in human cancer, which is critical for the cancer-therapy efficacy of lanperisone. ${ }^{56}$ In addition, constitutive activation of Nrf2 in lung cancer cells promotes tumorigenicity and contributes to resistance to carboplatin by up-regulating Nrf2-regulated genes involved in the increase of antioxidant capability, drug efflux, and detoxification. ${ }^{57}$ Oppositely, the inhibition of Nrf2 expression by RNA interference in lung cancer cells induces ROS generation and subsequently suppresses tumor growth, resulting in increased sensitivity to carboplatin-induced cell death in vitro and in vivo. ${ }^{57}$ Furthermore, endogenous antioxidants have been suggested as new cancer-therapy targets and are actively studied for this purpose. ${ }^{33,58,59}$ Therefore, antioxidants or the increase of antioxidative capability may potentially have an adverse effect on the efficacy of cancer treatment.

Iglesias-Bartolome el al, ${ }^{35}$ however, elegantly highlighted the fact that normal and cancer cells employ different signaling pathways as well as stress responses. More importantly, the study suggested that this difference between normal and cancer cells can provide a good strategy to reduce cancer therapy-induced side effects without compromising the efficacy of cancer therapy. Induction of Mn-SOD by rapamycin prevents radiation-induced $\mathrm{p} 16^{\mathrm{INK}}$ increase and depletion of tissue-repopulating stem cells, subsequently reducing the appearance of ulcers and mucositis. ${ }^{35}$ However, rapamycin treatment does not protect cancer cells from radiation-induced cell death, due to the inability of rapamycin to increase MnSOD in cancer cells. ${ }^{35}$ The authors suggested that because $p 16^{I N K}$ mutation (inactivation) is commonly found in head and neck squamous carcinoma, rapamycin has no impact on the activation of $\mathrm{p} 16^{\mathrm{INK}}$-dependent cell-senescence pathways in this cancer. ${ }^{35} \mathrm{D}$-Methionine also selectively protects normal cells but not cancer cells from radiation-induced cell death in vitro, and reduces radiation-induced mucosal injury without altering tumor response to the therapy in vivo. ${ }^{60} \mathrm{D}$-Methionine treatment protects normal keratinocytes from mitochondrial membrane loss induced by ionizing radiation, whereas no significant protection of the mitochondria membrane is observed in cancer cells with D-methionine treatment. ${ }^{60}$ However, the 
molecular mechanisms responsible for these differences were not determined in the study. Therefore, it has been suggested that the same agent may act differently on normal and cancer cells, which may be used as an effective strategy to manage mucositis without compromising cancer treatment.

In addition, many antioxidants have functions other than scavenging ROS. For example, vitamin E analogs are potent antioxidants and have been thought to mediate radioprotection by scavenging ROS. ${ }^{61}$ However, recent research has suggested that their radioprotective effect is elicited by the increase in the level of growth factors, such as granulocytecolony stimulating factor. ${ }^{61}$ Therefore, antioxidants or agents that induce antioxidant activity should be carefully used for mucositis management so that they selectively protect normal cells from cancer treatment. It is also important to identify the molecular difference between normal and cancer cells that makes the agents selectively protect normal but not cancer cells and to determine the agents' mechanisms of action beyond scavenging ROS within different contexts of normal and cancer cells.

\section{Inhibition of inflammation and cytokine production/secretion}

Inflammatory cytokines have been considered to play a critical role in the development of mucositis induced by chemotherapy and radiotherapy. ${ }^{20,62-64}$ Particularly, TNF $\alpha$, IL-1 $\beta$, and IL-6 $6^{7,62}$ have been implicated in mucositis and have been the targets of inhibition.

IL-1 $\beta$ is responsible for mucositis induced by the gutspecific deletion of $\beta$-transducin repeat-containing protein, an E3 ubiquitin ligase. ${ }^{65}$ Noticeably, IL- $1 \beta$ is derived from epithelial cells rather than from inflammatory cells after DNA damage via an unknown mechanism, and the secreted IL-1 $\beta$ causes mucositis by disrupting epithelial tight junctions. ${ }^{65}$ In addition, IL-1 $\beta$ and IL-18 mediate mucositis induced by irinotecan, a topoisomerase 1 inhibitor, evidenced by the inhibition of mucosal damage, inflammatory cell infiltration, and ulceration after the use of IL-1 receptor antagonists or the deletion of $I L-18 .{ }^{65} \mathrm{IL}-1 \mathrm{RA}$ is a secreted molecule that binds IL-1 receptors and acts as a natural antagonist of IL-1. ${ }^{66}$ IL-1RA effectively reduces intestinal mucositis induced by 5 -FU. ${ }^{67}$ Administration of recombinant IL-1RA after chemotherapy also reduces the acute lethal toxicity and intestinal mucosal damage induced by 5-FU and enhances intestinal recovery. ${ }^{67,68}$ Therefore, studies have demonstrated that IL-1 $\beta$ is critical in the development of intestinal mucositis and inhibition of IL-1 $\beta$ relieves mucositis induced by DNA damage.
Studies have also reported the application of commonly utilized pharmacological agents to treat mucositis, due to their inhibitory effects on cytokine production and/or secretion. Pentoxifylline, a methylated xanthine derivative, has been used for the treatment of peripheral vascular disease, and has been found to be a potent inhibitor of TNF $\alpha$ secretion. ${ }^{69,70}$ Pentoxifylline reduced oral mucositis induced by the administration of 5-FU followed by mechanical trauma to the cheek pouch in hamsters, ${ }^{71}$ as well as intestinal mucositis induced by irinotecan in mice. ${ }^{72}$ Minocycline, a tetracycline derivative, is a widely utilized antibiotic and has been demonstrated to have multiple functions, including the suppression of proinflammatory cytokines (eg, TNF $\alpha$ and IL-1 $\beta)^{73,74}$ and the inhibition of apoptosis pathways. ${ }^{75}$ Minocycline treatment mitigates intestinal mucositis induced by both 5-FU and irinotecan, which is attributed to minocycline's inhibitory effect on IL-1 $\beta$ and TNF $\alpha$ in small-intestine tissues and subsequent reduction in intestinal apoptosis. ${ }^{76}$ Furthermore, minocycline enhances the antitumor effects of 5-FU in mice xenografts of mouse colon cancer cells. ${ }^{76}$ A pilot study in hematopoietic cancer patients suggested that topical application of sesame oil, which has both antioxidative and anti-inflammatory activities, is useful for retardation of chemotherapy-induced oral mucositis. ${ }^{77}$ In the same study, cytological examination further demonstrated that inflammation induced by chemotherapy is reduced by sesame-oil application. ${ }^{77}$ Therefore, agents with anti-inflammatory activity effectively inhibit mucositis probably by inhibiting the production/secretion of the cytokines, IL-1 $\beta$ and TNF $\alpha$, which occurs early in chemotherapy-induced mucositis. ${ }^{78}$ However, these antiinflammatory agents may have other functions that may also contribute to the inhibition of mucositis. Since not all anti-inflammatory agents are effective in preventing the development of mucositis, ${ }^{79}$ it is important to identify their mechanisms of action using specific inhibitors to select effective agents for mucositis among many available antiinflammatory compounds derived from foods or traditional medicines.

$N F-\kappa B$ is a family of major genes activated by chemotherapy and radiotherapy. ${ }^{7,80} \mathrm{NF}-\kappa \mathrm{B}$ is a central regulator of genes induced by 5-FU, and the expression of NF- $\mathrm{KB}-$ regulated genes correlates with a mucositis-related phenomenon (ie, increase of proinflammatory cytokines). ${ }^{81}$ Accordingly, NF- $\mathrm{KB}$ has been considered to play a critical role in the upregulation of proinflammatory cytokines and the inflammation process in mucositis, ${ }^{7,79,81}$ and inhibition of NF- $\mathrm{KB}$ has been suggested as an attractive strategy for preventing mucositis. ${ }^{79}$ Most chemotherapeutic drugs induce 


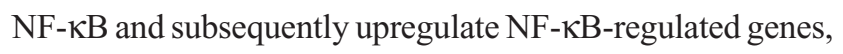
which have been suggested as the mechanism behind resistance to cancer therapy-induced apoptosis in cancer cells. ${ }^{82}$ Therefore, targeting NF- $\kappa \mathrm{B}$ could be a good strategy to treat cancer cells and protect normal cells from cancer treatment. Interestingly, turmeric, which has been demonstrated to have $\mathrm{NF}-\kappa \mathrm{B}$-inhibitory effects and therapeutic value in cancer treatment, ${ }^{82}$ has been shown to delay and reduce the severity of mucositis in head and neck cancer patients undergoing radiation therapy. ${ }^{83}$

However, a recent study demonstrated that NF- $\kappa \mathrm{B}$ activation is not involved in mucositis. ${ }^{65}$ The secreted IL- $1 \beta$ following DNA damage induces a mucosal barrier breach in an NF- $\kappa \mathrm{B}$-independent manner. ${ }^{65}$ Moreover, the tissue damage caused by mucosal barrier disruption is exacerbated in the absence of NF- $\mathrm{B}$, because of failure to express the endogenous IL- $1 \beta$ receptor antagonist IL-1RA, and thereby $\mathrm{NF}-\kappa \mathrm{B}$ inhibition exacerbates mucositis rather than inhibits the source of inflammation. ${ }^{65}$ Therefore, it needs to be reexamined whether NF- $\kappa \mathrm{B}$ mediates mucositis induced by chemotherapy and radiation or whether NF- $\kappa B$-related gene expression merely coincides with the mucositis phenomenon. The role of NF- $\kappa \mathrm{B}$ in mucositis needs to be further elucidated in the future.

\section{Inhibition of apoptosis}

It has been demonstrated that apoptosis is critical for the development of mucositis. ${ }^{84-87}$ When mice were treated with various cytotoxic agents, including radiation, antibiotics, and alkylating agents, all of the tested cytotoxic agents caused apoptosis within 12 hours of administration. ${ }^{84}$ Studies have also indicated that apoptosis is a critical event in the occurrence of 5-FU-induced intestinal mucositis, and many apoptotic cells are observed in intestinal crypts before serious mucosal destruction in mice and humans. ${ }^{87,88}$ In addition, a time-course study in cancer patients receiving various chemotherapy treatments demonstrated that the earliest effect (within 24 hours after treatment) is increased apoptosis in intestinal crypts. ${ }^{85}$ This increase in apoptosis by chemotherapy is preceded by a reduction in villus area, crypt length, mitotic counts, and enterocyte-cell height in human intestine. ${ }^{85}$

In rapidly proliferating tissues, such as the GIT, the stringent control of cell proliferation and cell death by apoptosis is critical to the maintenance of tissue homeostasis, and apoptosis plays an important role in controlling the number of stem cells. ${ }^{89,90}$ Many cytotoxic drugs, including DNAdamaging agents, cause apoptotic cell death at the bottom of the crypts where stem cells reside, whereas apoptosis typically occurs at the top of the crypts during the normal differentiation process, ${ }^{84,90}$ contributing to the loss of regeneration capability. The administration of CXCL9 attenuates the severity of intestinal mucositis induced by 5-FU and reduces structural damage to the intestinal mucosa. ${ }^{91}$ The protective effect of CXCL9 is attributed to the preservation of regenerative cells, such as stem/progenitor cells, against cell death induced by chemotherapy that targets cells in rapid proliferation (S phase). ${ }^{91}$ CXCL9 treatment decreases both proliferation and apoptosis in the intestinal crypt during chemotherapy, whereas it increases the proliferation rate of intestinal crypts after chemotherapy. ${ }^{91}$ Therefore, CXCL9 treatment preserves the regenerative cells available for mucosal repair, as demonstrated by its protective effect on hematopoietic stem/progenitor cells against chemotherapeutic drugs by arresting cells in the $\mathrm{G}_{0}$ phase. ${ }^{92}$

Apoptosis has been demonstrated to be an important event in oral mucositis induced by radiotherapy as well. The RNA-binding protein HuR undergoes cleavage by caspase 3 following irradiation in an oral mucositis mouse model, and subsequently promotes the expression of the proapoptotic factor Bax. ${ }^{86,93}$ Specific inhibition of caspase 3 by the smallmolecule compound NSC321205 increases the clonogenic capacity of primary oral keratinocytes and increases basal layer cellularity, leading to the elevation of epithelial cell growth in the tongues of mice with oral mucositis. ${ }^{86}$ This protective effect of NSC321205 is mediated by a decrease in caspase 3 activity and the consequent inhibition of $\mathrm{HuR}$ cleavage and Bax expression. ${ }^{86}$ The expression of proapoptotic proteins (eg, p53) is elevated, whereas the levels of antiapoptotic proteins (eg, Bcl-2 and Mcl-1) are reduced in smear preparations of normal-looking buccal mucosa or in mucosa adjacent to oral mucositis regions in patients that develop mucositis during radiotherapy for head and neck cancer. $^{94}$

Inflammatory molecules often mediate apoptosis in mucositis, and thus the suppression of inflammation attenuates chemotherapy-induced apoptosis, as well as the development of mucositis. The $5-\mathrm{HT}_{3}$-receptor antagonists ramosetron and ondansetron ameliorate 5-FU-induced intestinal mucositis in mice, and this effect is attributed to the suppression of apoptotic responses in the intestinal crypt cells via the inhibition of cytokine expression. ${ }^{95}$ In humans, 5-HT is primarily synthesized and localized in the enterochromaffin cells of the GI mucosa, ${ }^{96}$ and its plasma levels have been shown to be increased by chemotherapy, such as 5-FU. ${ }^{95}$ Ramosetron and ondansetron reduce the secretion of TNF $\alpha$ and the activation of apoptosis induced by 5 -FU within 24 hours posttreatment. They also suppress 
the shortening of villi and the destruction of intestinal crypts in a dose-dependent manner. ${ }^{95}$

Saireito, a traditional Japanese herbal medicine, is a combined formulation of two herbal medicines used to treat inflammatory diseases. ${ }^{78,97}$ Saireito treatment attenuates intestinal mucositis induced by $5-\mathrm{FU}$ in a process mediated by the inhibition of TNF $\alpha$ and IL- $1 \beta$ expression, contributing to the suppression of 5-FU-induced apoptosis without affecting cell proliferation. ${ }^{78}$ Therefore, apoptosis is a critical event in the development of mucositis induced by radiotherapy and chemotherapy. Inhibition of apoptosis in the early stage of cancer treatment reduces the likelihood of mucositis development and/or the severity of mucositis whether the induction of apoptosis is mediated through cytokines or not.

However, conventional chemotherapy and radiotherapy act by inducing apoptosis. This cancer therapy-induced apoptosis also occurs in normal cells, causing mucositis. Therefore, agents developed for the prevention of mucositis should protect normal cells from cancer therapy-induced apoptotic cell death without impeding cancer therapyinduced apoptosis in cancer cells. Encouragingly, 5-FU efficacy is not attenuated by daily administration of either saireito $^{78}$ or ramosetron ${ }^{95}$ in a mouse model of colon cancer cell (Colon 38)-derived tumor implants, although underlying mechanisms are unknown. In addition, it is also important that the agent used for preventing mucositis is able to selectively preserve the GIT stem/progenitor cells with the ability to regenerate and restore the epithelial structure and integrity upon the cessation of cancer treatment.

\section{Conclusion}

Mucositis affects most patients undergoing chemotherapy and radiotherapy and is a major clinical and economic burden that severely affects patient survival and quality of life. ${ }^{1}$ However, management of mucositis largely involves the control of symptoms using antibiotics, anesthetics, and analgesics, ${ }^{8,13}$ and there are very limited therapeutic agents available for mucositis treatment. ${ }^{79,98}$ Mucositis is the result of complex biological events involving a series of signaling pathways and interactions between mucosa and submucosa. ${ }^{1,13}$ Mucositis can be inhibited by the modulation of radiotherapyor chemotherapy-induced pathways independently of cancer treatment, which provides an opportunity for the development of more targeted therapies and interventions. ${ }^{13,21}$ Agents that stimulate the growth or migration of epithelial cells are likely unsuitable for mucositis management, since they can exert the same mitotic and migratory effect on cancer cells. ${ }^{79}$

Figure 1 describes the pathways that have been shown to be effective for treating mucositis using potential therapeutic agents for mucositis management. The pathways are related to early events in the pathogenesis of mucositis, and targeting these pathways may provide a good strategy to effectively prevent mucositis. These early events include ROS scavenging, inhibition of specific cytokine production or inflammation, and inhibition of apoptosis. Chemotherapy and radiotherapy induce ROS generation and apoptosis induction shortly after (within 24 hours) administration. Protection from cancer therapy-induced ROS and apoptosis may effectively prevent mucositis. However, many cancer chemotherapy and radiotherapy agents exert their therapeutic effect by the generation of ROS and the induction of apoptosis. Since both cancer-therapy agents and therapeutic agents for mucositis treatment act on both normal cells and cancer cells, agents for the inhibition of mucositis should be effective through a mechanism that selectively protects normal cells without compromising cancer treatment. Antiinflammation may be a good strategy, since inflammation is also related to the progression of cancer. In addition, anti-inflammation is beneficial in that it is also induced in submucosa and this inflammatory signal from submucosa further accelerates developing mucositis. ${ }^{28}$ However, not all anti-inflammatory agents are effective for mucositis treatment. ${ }^{79}$ Therefore, the exact mechanism must be understood to identify effective agents among many available anti-inflammatory compounds derived from foods or common pharmaceuticals. Although many agents acting on aforementioned pathways have not been addressed by MASCC/ISSO clinical practice guidelines due to inadequate and/or conflicting evidence, ${ }^{47}$ it is encouraging that some agents (eg, vitamin E and NAC) have been suggested to be effective in the clinical setting as well.

\section{Acknowledgments}

The study was supported by a grant from the National Research Foundation of Korea funded by the Ministry of Science, ICT, and Future Planning (2014R1A1A3050916).

\section{Disclosure}

The author reports no conflicts of interest in this work.

\section{References}

1. Sonis ST. The pathobiology of mucositis. Nat Rev Cancer. 2004; 4(4):277-284.

2. Epstein JB, Schubert MM. Oropharyngeal mucositis in cancer therapy: review of pathogenesis, diagnosis, and management. Oncology (Williston Park). 2003;17(12):1767-1779; discussion 1779-1782, 1791-1792.

3. Sonis ST. Oral mucositis in cancer therapy. J Support Oncol. 2004; 2(6 Suppl 3):3-8.

4. Barasch A, Peterson DE. Risk factors for ulcerative oral mucositis in cancer patients: unanswered questions. Oral Oncol. 2003;39(2): 91-100. 
5. Schwab M, Zanger UM, Marx C, et al. Role of genetic and nongenetic factors for fluorouracil treatment-related severe toxicity: a prospective clinical trial by the German 5-FU Toxicity Study Group. J Clin Oncol. 2008;26(13):2131-2138.

6. Köstler WJ, Hejna M, Wenzel C, Zielinski CC. Oral mucositis complicating chemotherapy and/or radiotherapy: options for prevention and treatment. CA Cancer J Clin. 2001;51(5):290-315.

7. Logan RM, Stringer AM, Bowen JM, et al. The role of pro-inflammatory cytokines in cancer treatment-induced alimentary tract mucositis: pathobiology, animal models and cytotoxic drugs. Cancer Treat Rev. 2007;33(5):448-460.

8. Pico JL, Avila-Garavito A, Naccache P. Mucositis: its occurrence, consequences, and treatment in the oncology setting. Oncologist. 1998;3(6):446-451.

9. Naidu MU, Ramana GV, Rani PU, Mohan IK, Suman A, Roy P. Chemotherapy-induced and/or radiation therapy-induced oral mucositis - complicating the treatment of cancer. Neoplasia. 2004;6(5): 423-431.

10. Knox JJ, Puodziunas AL, Feld R. Chemotherapy-induced oral mucositis: prevention and management. Drugs Aging. 2000;17(4):257-267.

11. Peterson DE, Bensadoun RJ, Roila F. Management of oral and gastrointestinal mucositis: ESMO Clinical Practice Guidelines. Ann Oncol. 2010;21 Suppl 5:v261-v265.

12. Parkhill A. Oral mucositis and stomatitis associated with conventional and targeted anticancer therapy. J Pharmacovigil. 2013;1(4): 1000112.

13. Sonis ST. Mucositis: the impact, biology and therapeutic opportunities of oral mucositis. Oral Oncol. 2009;45(12):1015-1020.

14. Watters AL, Epstein JB, Agulnik M. Oral complications of targeted cancer therapies: a narrative literature review. Oral Oncol. 2011; 47(6):441-448.

15. Li E, Trovato JA. New developments in management of oral mucositis in patients with head and neck cancer or receiving targeted anticancer therapies. Am J Health Syst Pharm. 2012;69(12):1031-1037.

16. O’Donnell A, Faivre S, Burris HA 3rd, et al. Phase I pharmacokinetic and pharmacodynamic study of the oral mammalian target of rapamycin inhibitor everolimus in patients with advanced solid tumors. $J$ Clin Oncol. 2008;26(10):1588-1595.

17. Sankhala K, Mita A, Kelly K, Mahalingam D, Giles F, Mita M. The emerging safety profile of mTOR inhibitors, a novel class of anticancer agents. Target Oncol. 2009;4(2):135-142.

18. Elting LS, Chang YC, Parelkar P, et al. Risk of oral and gastrointestinal mucosal injury among patients receiving selected targeted agents: a meta-analysis. Support Care Cancer. 2013;21(11):3243-3254.

19. Sonis S, Treister N, Chawla S, Demetri G, Haluska F. Preliminary characterization of oral lesions associated with inhibitors of mammalian target of rapamycin in cancer patients. Cancer. 2010;116(1):210-215.

20. Sultani M, Stringer AM, Bowen JM, Gibson RJ. Anti-inflammatory cytokines: important immunoregulatory factors contributing to chemotherapy-induced gastrointestinal mucositis. Chemother Res Pract. 2012;2012:490804.

21. Keefe DM, Sonis ST, Bowen JM. Emerging drugs for chemotherapy-induced mucositis. Expert Opin Emerg Drugs. 2008;13(3): $511-522$.

22. Sonis ST. Mucositis as a biological process: a new hypothesis for the development of chemotherapy-induced stomatotoxicity. Oral Oncol. 1998;34(1):39-43

23. Conklin KA. Chemotherapy-associated oxidative stress: impact on chemotherapeutic effectiveness. Integr Cancer Ther. 2004;3(4):294-300.

24. Valerie K, Yacoub A, Hagan MP, et al. Radiation-induced cell signaling: inside-out and outside-in. Mol Cancer Ther. 2007;6(3):789-801.

25. Yamamori T, Yasui H, Yamazumi M, et al. Ionizing radiation induces mitochondrial reactive oxygen species production accompanied by upregulation of mitochondrial electron transport chain function and mitochondrial content under control of the cell cycle checkpoint. Free Radic Biol Med. 2012;53(2):260-270.
26. Yoshino F, Yoshida A, Nakajima A, Wada-Takahashi S, Takahashi SS, Lee MC. Alteration of the redox state with reactive oxygen species for 5-fluorouracil-induced oral mucositis in hamsters. PloS One. 2013;8(12):e82834.

27. Schroeder KW. Role of mesalazine in acute and long-term treatment of ulcerative colitis and its complications. Scand J Gastroenterol Suppl. 2002;(236):42-47.

28. Bamba S, Andoh A, Yasui H, Araki Y, Bamba T, Fujiyama Y. Matrix metalloproteinase-3 secretion from human colonic subepithelial myofibroblasts: role of interleukin-17. J Gastroenterol. 2003;38(6):548-554.

29. Ewald JA, Desotelle JA, Wilding G, Jarrard DF. Therapy-induced senescence in cancer. J Natl Cancer Inst. 2010;102(20):1536-1546.

30. Coppé JP, Desprez PY, Krtolica A, Campisi J. The senescence-associated secretory phenotype: the dark side of tumor suppression. Annu Rev Pathol. 2010;5:99-118.

31. Ha HC, Thiagalingam A, Nelkin BD, Casero RA Jr. Reactive oxygen species are critical for the growth and differentiation of medullary thyroid carcinoma cells. Clin Cancer Res. 2000;6(9):3783-3787.

32. Sauer H, Wartenberg M, Hescheler J. Reactive oxygen species as intracellular messengers during cell growth and differentiation. Cell Physiol Biochem. 2001;11(4):173-186.

33. Glasauer A, Chandel NS. Targeting antioxidants for cancer therapy. Biochem Pharmacol. 2014;92(1):90-101.

34. Guo H, Seixas-Silva JA Jr, Epperly MW, et al. Prevention of radiationinduced oral cavity mucositis by plasmid/liposome delivery of the human manganese superoxide dismutase (SOD2) transgene. Radiat Res. 2003;159(3):361-370.

35. Iglesias-Bartolome R, Patel V, Cotrim A, et al. mTOR inhibition prevents epithelial stem cell senescence and protects from radiationinduced mucositis. Cell Stem Cell. 2012;11(3):401-414.

36. Murphy CK, Fey EG, Watkins BA, Wong V, Rothstein D, Sonis ST. Efficacy of superoxide dismutase mimetic M40403 in attenuating radiation-induced oral mucositis in hamsters. Clin Cancer Res. 2008; 14(13):4292-4297.

37. Takano H, Momota Y, Kani K, et al. $\gamma$-Tocotrienol prevents 5-FUinduced reactive oxygen species production in human oral keratinocytes through the stabilization of 5-FU-induced activation of $\mathrm{Nrf} 2$. Int J Oncol. 2015;46(4):1453-1460.

38. Ucuncu H, Ertekin MV, Yoruk O, et al. Vitamin E and L-carnitine, separately or in combination, in the prevention of radiation-induced oral mucositis and myelosuppression: a controlled study in a rat model. J Radiat Res. 2006;47(1):91-102.

39. Azizi A, Alirezaei S, Pedram P, Mafi RA. Efficacy of topical and systemic vitamin $\mathrm{E}$ in preventing chemotherapy-induced oral mucositis. Rep Radiother Oncol. 2015;2(1):15-18.

40. El-Housseiny AA, Saleh SM, El-Masry AA, Allam AA. The effectiveness of vitamin "E" in the treatment of oral mucositis in children receiving chemotherapy. J Clin Pediatr Dent. 2007;31(3): $167-170$

41. Ghoreishi Z, Shidfar F, Iravani M, Esfahani A, Ghavamzadeh A. Effect of vitamin $\mathrm{E}$ on chemotherapy-induced mucositis and neutropenia in leukemic patients undergoing bone marrow transplantation. Asia Pac J Clin Oncol. 2007;3(3):113-118.

42. Moslehi A, Taghizadeh-Ghehi M, Gholami K, et al. N-acetyl cysteine for prevention of oral mucositis in hematopoietic SCT: a doubleblind, randomized, placebo-controlled trial. Bone Marrow Transplant. 2014;49(6):818-823.

43. Geronikaki AA, Gavalas AM. Antioxidants and inflammatory disease: synthetic and natural antioxidants with anti-inflammatory activity. Comb Chem High Throughput Screen. 2006;9(6):425-442.

44. Valencia J, Velilla C, Urpegui A, et al. The efficacy of orgotein in the treatment of acute toxicity due to radiotherapy on head and neck tumors. Tumori. 2002;88(5):385-389.

45. Dostert C, Petrilli V, Van Bruggen R, Steele C, Mossman BT, Tschopp J. Innate immune activation through Nalp3 inflammasome sensing of asbestos and silica. Science. 2008;320(5876):674-677. 
46. Arifa RD, Madeira MF, de Paula TP, et al. Inflammasome activation is reactive oxygen species dependent and mediates irinotecan-induced mucositis through IL-1 $\beta$ and IL-18 in mice. Am J Pathol. 2014; 184(7):2023-2034.

47. Lalla RV, Bowen J, Barasch A, et al. MASCC/ISOO clinical practice guidelines for the management of mucositis secondary to cancer therapy. Cancer. 2014;120(10):1453-1461.

48. Arbabi-Kalati F, Arbabi-Kalati F, Deghatipour M, Ansari Moghadam A. Evaluation of the efficacy of zinc sulfate in the prevention of chemotherapy-induced mucositis: a double-blind randomized clinical trial. Arch Iran Med. 2012;15(7):413-417.

49. Lin LC, Que J, Lin LK, Lin FC. Zinc supplementation to improve mucositis and dermatitis in patients after radiotherapy for head-andneck cancers: a double-blind, randomized study. Int J Radiat Oncol Biol Phys. 2006;65(3):745-750.

50. Powell SR. The antioxidant properties of zinc. J Nutr. 2000; 130(5S Suppl):1447S-1454S.

51. Block KI. Antioxidants and cancer therapy: furthering the debate. Integr Cancer Ther. 2004;3(4):342-348.

52. Ozben T. Antioxidant supplementation on cancer risk and concurrent use of antioxidants during cancer therapy: an update. Curr Top Med Chem. Epub 2014 Dec 29.

53. Bairati I, Meyer F, Gelinas M, et al. Randomized trial of antioxidant vitamins to prevent acute adverse effects of radiation therapy in head and neck cancer patients. J Clin Oncol. 2005;23(24):5805-5813.

54. Bairati I, Meyer F, Gelinas M, et al. A randomized trial of antioxidant vitamins to prevent second primary cancers in head and neck cancer patients. J Natl Cancer Inst. 2005;97(7):481-488.

55. Sayin VI, Ibrahim MX, Larsson E, Nilsson JA, Lindahl P, Bergo MO. Antioxidants accelerate lung cancer progression in mice. Sci Transl Med. 2014;6(221):221 ra15.

56. Shaw AT, Winslow MM, Magendantz M, et al. Selective killing of K-ras mutant cancer cells by small molecule inducers of oxidative stress. Proc Natl Acad Sci U S A. 2011;108(21):8773-8778.

57. Singh A, Boldin-Adamsky S, Thimmulappa RK, et al. RNAimediated silencing of nuclear factor erythroid-2-related factor 2 gene expression in non-small cell lung cancer inhibits tumor growth and increases efficacy of chemotherapy. Cancer Res. 2008;68(19): 7975-7984.

58. Koháryová M, Kollárová M. Thioredoxin system - a novel therapeutic target. Gen Physiol Biophys. 2015;34(3):221-233.

59. Mahmood DF, Abderrazak A, El Hadri K, Simmet T, Rouis M. The thioredoxin system as a therapeutic target in human health and disease. Antioxid Redox Signal. 2013;19(11):1266-1303.

60. Vuyyuri SB, Hamstra DA, Khanna D, et al. Evaluation of D-methionine as a novel oral radiation protector for prevention of mucositis. Clin Cancer Res. 2008;14(7):2161-2170.

61. Singh VK, Beattie LA, Seed TM. Vitamin E: tocopherols and tocotrienols as potential radiation countermeasures. J Radiat Res. 2013; 54(6):973-988.

62. Ong ZY, Gibson RJ, Bowen JM, et al. Pro-inflammatory cytokines play a key role in the development of radiotherapy-induced gastrointestinal mucositis. Radiat Oncol. 2010;5:22.

63. Pusztai L, Mendoza TR, Reuben JM, et al. Changes in plasma levels of inflammatory cytokines in response to paclitaxel chemotherapy. Cytokine. 2004;25(3):94-102.

64. Sonis S. The quest for effective treatments of mucositis. J Support Oncol. 2011;9(5):170-171.

65. Kanarek N, Grivennikov SI, Leshets M, et al. Critical role for IL-1 in DNA damage-induced mucositis. Proc Natl Acad Sci US A. 2014; 111(6):E702-E711.

66. Arend WP, Guthridge CJ. Biological role of interleukin 1 receptor antagonist isoforms. Ann Rheum Dis. 2000;59 Suppl 1:i60-i64.

67. Wu Z, Han X, Qin S, et al. Interleukin 1 receptor antagonist reduces lethality and intestinal toxicity of 5-fluorouracil in a mouse mucositis model. Biomed Pharmacother. 2011;65(5):339-344.
68. Wu Z, Han $\mathrm{X}$, Qin $\mathrm{S}$, et al. Interleukin 1 receptor antagonist reduces lethality and intestinal toxicity of 5-fluorouracil in a mouse mucositis model. Biomed Pharmacother. 2010;64(9):589-593.

69. Blaine TA, Pollice PF, Rosier RN, Reynolds PR, Puzas JE, O’Keefe RJ. Modulation of the production of cytokines in titanium-stimulated human peripheral blood monocytes by pharmacological agents: the role of cAMP-mediated signaling mechanisms. J Bone Joint Surg Am. 1997;79(10):1519-1528.

70. Marques LJ, Zheng L, Poulakis N, Guzman J, Costabel U. Pentoxifylline inhibits TNF- $\alpha$ production from human alveolar macrophages. Am $J$ Respir Crit Care Med. 1999;159(2):508-511.

71. Lima V, Brito GA, Cunha FQ, et al. Effects of the tumour necrosis factor- $\alpha$ inhibitors pentoxifylline and thalidomide in short-term experimental oral mucositis in hamsters. Eur J Oral Sci. 2005; 113(3):210-217.

72. Melo ML, Brito GA, Soares RC, et al. Role of cytokines (TNF- $\alpha$, IL-1 $\beta$ and $\mathrm{KC}$ ) in the pathogenesis of CPT-11-induced intestinal mucositis in mice: effect of pentoxifylline and thalidomide. Cancer Chemother Pharmacol. 2008;61(5):775-784.

73. Cata JP, Weng HR, Dougherty PM. The effects of thalidomide and minocycline on taxol-induced hyperalgesia in rats. Brain Res. 2008;1229:100-110.

74. Sriram K, Miller DB, O’Callaghan JP. Minocycline attenuates microglial activation but fails to mitigate striatal dopaminergic neurotoxicity: role of tumor necrosis factor- $\alpha$. J Neurochem. 2006;96(3):706-718.

75. Zhu S, Stavrovskaya IG, Drozda M, et al. Minocycline inhibits cytochrome $\mathrm{C}$ release and delays progression of amyotrophic lateral sclerosis in mice. Nature. 2002;417(6884):74-78.

76. Huang TY, Chu HC, Lin YL, et al. Minocycline attenuates 5-fluorouracilinduced small intestinal mucositis in mouse model. Biochem Biophys Res Commun. 2009;389(4):634-639.

77. Okada S, Sudo A, Nishio J. Topical application of sesame oil for the prevention of chemotherapy-induced oral mucositis: pilot study in seven hematopoietic cancer patients. Int J Nurs Clin Pract. 2015;2:123.

78. Kato S, Hayashi S, Kitahara Y, et al. Saireito (TJ-114), a Japanese traditional herbal medicine, reduces 5-fluorouracil-induced intestinal mucositis in mice by inhibiting cytokine-mediated apoptosis in intestinal crypt cells. PloS One. 2015;10(1):e0116213.

79. Bian L, Han G, Zhao CW, Garl PJ, Wang XJ. The role of Smad7 in oral mucositis. Protein Cell. 2015;6(3):160-169.

80. Sonis ST. The biologic role for nuclear factor- $\kappa \mathrm{B}$ in disease and its potential involvement in mucosal injury associated with anti-neoplastic therapy. Crit Rev Oral Biol Med. 2002;13(5):380-389.

81. Chang CT, Ho TY, Lin H, et al. 5-Fluorouracil induced intestinal mucositis via nuclear factor- $\kappa \mathrm{B}$ activation by transcriptomic analysis and in vivo bioluminescence imaging. PloS One. 2012;7(3):e31808.

82. Kwon Y. Curcumin as a cancer chemotherapy sensitizing agent. J Korean Soc Appl Biol Chem. 2014;57(2):273-280.

83. Rao S, Dinkar C, Vaishnav LK, et al. The Indian spice turmeric delays and mitigates radiation-induced oral mucositis in patients undergoing treatment for head and neck cancer: an investigational study. Integr Cancer Ther. 2013;13(3):201-210.

84. Ijiri K, Potten CS. Further studies on the response of intestinal crypt cells of different hierarchical status to eighteen different cytotoxic agents. Br J Cancer. 1987;55(2):113-123.

85. Keefe DM, Brealey J, Goland GJ, Cummins AG. Chemotherapy for cancer causes apoptosis that precedes hypoplasia in crypts of the small intestine in humans. Gut. 2000;47(5):632-637.

86. Talwar S, House R, Sundaramurthy S, Balasubramanian S, Yu H, Palanisamy $\mathrm{V}$. Inhibition of caspases protects mice from radiationinduced oral mucositis and abolishes the cleavage of RNA-binding protein HuR. J Biol Chem. 2014;289(6):3487-3500.

87. Yasuda M, Kato S, Yamanaka N, et al. Potential role of the NADPH oxidase NOX1 in the pathogenesis of 5-fluorouracil-induced intestinal mucositis in mice. Am J Physiol Gastrointest Liver Physiol. 2012;302(10):G1133-G1142. 
88. Anilkumar TV, Sarraf CE, Hunt T, Alison MR. The nature of cytotoxic drug-induced cell death in murine intestinal crypts. Br J Cancer. 1992;65(4):552-558.

89. Potten CS, Wilson JW, Booth C. Regulation and significance of apoptosis in the stem cells of the gastrointestinal epithelium. Stem Cells. 1997; 15(2):82-93.

90. Kwon Y, Magnuson BA. Aging alters acute apoptotic response to azoxymethane in the colon of rats. Exp Gerontol. 2007;42(12): 1154-1161.

91. Han X, Wu Z, Di J, et al. CXCL9 attenuated chemotherapy-induced intestinal mucositis by inhibiting proliferation and reducing apoptosis. Biomed Pharmacother. 2011;65(8):547-554.

92. Lu H, Zhu S, Qian L, et al. Activated expression of the chemokine Mig after chemotherapy contributes to chemotherapy-induced bone marrow suppression and lethal toxicity. Blood. 2012;119(21):4868-4877.

93. Feldman T, Kabaleeswaran V, Jang SB, et al. A class of allosteric caspase inhibitors identified by high-throughput screening. Mol Cell. 2012;47(4):585-595.
94. Xanthinaki A, Nicolatou-Galitis O, Athanassiadou P, et al. Apoptotic and inflammation markers in oral mucositis in head and neck cancer patients receiving radiotherapy: preliminary report. Support Care Cancer. 2008;16(9):1025-1033.

95. Yasuda M, Kato S, Yamanaka N, et al. 5- $\mathrm{HT}_{3}$ receptor antagonists ameliorate 5-fluorouracil-induced intestinal mucositis by suppression of apoptosis in murine intestinal crypt cells. Br J Pharmacol. $2013 ; 168(6): 1388-1400$.

96. Talley NJ. Serotoninergic neuroenteric modulators. Lancet. 2001; 358(9298):2061-2068.

97. Ito T, Seo N, Yagi H, et al. Unique therapeutic effects of the JapaneseChinese herbal medicine, sairei-to, on Th1/Th2 cytokines balance of the autoimmunity of MRL/lpr mice. J Dermatol Sci. 2002; 28(3):198-210.

98. Blijlevens N, Sonis S. Palifermin (recombinant keratinocyte growth factor-1): a pleiotropic growth factor with multiple biological activities in preventing chemotherapy- and radiotherapy-induced mucositis. Ann Oncol. 2007;18(5):817-826.
OncoTargets and Therapy

\section{Publish your work in this journal}

OncoTargets and Therapy is an international, peer-reviewed, open access journal focusing on the pathological basis of all cancers, potential targets for therapy and treatment protocols employed to improve the management of cancer patients. The journal also focuses on the impact of management programs and new therapeutic agents and protocols on

\section{Dovepress}

patient perspectives such as quality of life, adherence and satisfaction. The manuscript management system is completely online and includes a very quick and fair peer-review system, which is all easy to use. Visit http://www.dovepress.com/testimonials.php to read real quotes from published authors. 